Part of the Journal of Research of the National Bureau of Standards

\title{
Acid-Base Reactions in Organic Solvents. Behavior of Some Halogenated Derivatives of Phenolsulfone- phthalein with Different Classes of Organic Bases in Benzene
}

\author{
By Marion Maclean Davis, Priscilla J. Schuhmann, and Mary Ellen Lovelace
}

\begin{abstract}
This paper, the second in a series concerning the use of indicator dyes to study the reactions of organic acids and bases in organic solvents, deals with halogen derivatives of phenolsulfonephthalein. Spectrophotometric data are given for mixtures of bromocresol green, bromophenol blue, iodophenol blue, and tetrabromophenol blue with primary, secondary, and tertiary aliphatic amines in benzene; and qualitative data are tabulated for chlorophenol blue, bromochlorophenol blue, chlorophenol red, bromophenol red, bromocresol purple, and bromothymol blue. Comparisons are made of the phenolsulfonephthaleins and the bromophthalein magentas. The structural changes that accompany the color phenomena are discussed. Suggestions are made regarding the use of the indicators in inert solvents.
\end{abstract}

\section{Introduction}

A previous article $[1]^{1}$ described in detail the preparation of the acidic indicators, bromophthalein magenta $\mathrm{E}$ and bromophthalein magenta B (respectively, the ethyl ester and the $n$-butyl ester of tetrabromophenolphthalein), and their reaction with various types of organic derivatives of ammonia in benzene and in other organic solvents. The objectives of our work on the application of indicator dyes to the measurement of acidity and basicity in organic solvents were discussed, and a review of theoretical aspects of acid-base reactions in organic media and of experimental contributions of several other investigators to this field was also presented.

This article deals with the reactions of 10 halogen derivatives of phenolsulfonephthalein with different types of organic bases in benzene. The phenolsulfonephthaleins are dibasic acids. They are readily available commercially and are widely used as acid-base indicators in aqueous media, because they undergo vivid color changes at definite $\mathrm{pH}$ values. Their behavior in benzene is more complex. In particular, the change that

${ }_{1}^{1}$ Figures in brackets indicate the literature references at the end of this paper. occurs during the second stage of neutralization depends upon the nature of the base that is added. It is different for primary, secondary, and tertiary aliphatic amines. These differentiating reactions parallel those previously described for bromophthalein magenta [1]. The reactions of the phenolsulfonephthaleins were investigated less thoroughly than those of the bromophthalein magentas, because various aspects of their behavior are predictable from their structural relationship to the bromophthalein magentas, and also because the sulfonephthaleins are in general less suitable acid-base indicators for organic solvents than the bromophthalein magentas. The structures and reactions of the two groups of indicator dyes are compared in sections to follow, but reference to the first paper in this series is essential for complete details of the reactions of the bromophthalein magentas and for a more extensive interpretation of the role of the solvent in acid-base reactions.

\section{Apparatus and Method}

The spectrophotometric data were obtained with a Beckman quartz photoelectric spectrophotometer $[2,3]$ in which a pair of stoppered cells and a specially designed cell compartment were sub- 
stituted for the cells and cell holder supplied with the commercial instrument. Each cell consisted of a pair of optically matched crystalline quartz endplates $2 \mathrm{~mm}$ in thickness and a stoppered quartz cylinder $3.8 \mathrm{~cm}$ in diameter and $1 \mathrm{~cm}$ in length, open and polished at the ends. The construction of the cell holder and the manner of assembling the parts of the cell were described previously [1].

In most of the measurements, one cell contained the pure solvent and the other cell contained the solution under investigation. Solutions were prepared in a room kept at $25^{\circ} \mathrm{C}$, and measurements were made at $26^{\circ} \pm 2^{\circ} \mathrm{C}$ [1]. Volumetric flasks and pipettes calibrated at the Bureau were used throughout. Stock solutions of known concentrations of the separate compounds were prepared and were diluted quantitatively immediately before use, to give the desired mixtures. The microbalance used for weighing the indicator dyes gave a precision of 0.5 to 1.0 percent.

Readings were usually made at intervals of 5 or $10 \mathrm{~m} \mu$. In a few cases additional readings were made at intervals of $1 \mathrm{~m} \mu$. Measurements of the percentage transmittancy [4] were usually reproducible within \pm 0.2 percent. In the figures to follow the transmittancies in percentages and wavelengths in millimicrons are plotted as ordinates and abscissas, respectively. Values for the molar absorbancy index, $a_{M}$, were calculated by means of the equation

$$
a_{M}=\frac{-\log _{10} T_{S}}{b \times M}
$$

in which $T_{s}=T_{\text {soln }} / T_{\text {solv }}=$ the transmittancy of the solute, $b$ is the depth of the cell in centimeters, and $M$ is the molar concentration of the solution. The absorbancy, often referred to as the "optical density," is the negative logarithm of the transmittancy [4].

\section{Materials}

The benzene used as the solvent and the organic bases were the same materials as those used in the preceding investigation [1].

In table 1 the indicators are listed under both common and chemical names, together with descriptive comments. The general structural for-

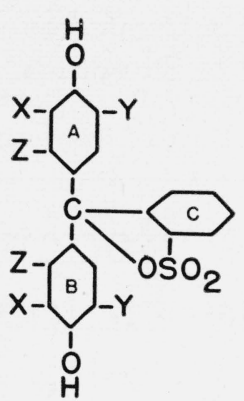

FIGURE 1. Structural formula for a phenolsulfonephthalein indicator

mula for phenolsulfonephthalein indicators is given in figure 1 . The nature of the substituents in positions $X, Y$, and $Z$ of rings $A$ and $B$ is shown in table 1. In tetrabromophenol blue, the four unlabeled positions in ring $C$ are occupied by bromine atoms; in all of the other sulfonephthaleins listed, by hydrogen atoms.

The indicators were obtained from several commercial sources. Bromophenol blue, chlorophenol blue, iodophenol blue, and tetrabromophenol blue were dried in a vacuum oven or Abderhalden apparatus before use. The remaining indicators were taken from freshly opened containers.

Microanalyses for the various indicators are presented in table 2. The sample of bromochlorophenol blue appeared to contain an acidic impurity and was therefore not analyzed. Two samples of chlorophenol red, from different commercial sources, were analyzed. Both samples showed a lower carbon content and higher chlorine content than the calculated values. Iodophenol blue showed too low carbon and too low iodine content, and tetrabromophenol blue, high carbon and hydrogen and low bromine. The percentage compositions found for the other six indicators are in satisfactory agreement with the theoretical values.

\section{Color Transformations of Phenolsulfonephthaleins}

An understanding of the relation between the color and the environment of phenolsulfonephthaleins is necessary to interpret experimental observations of their behavior in benzene. 
TABLE 1. List and description of indicators

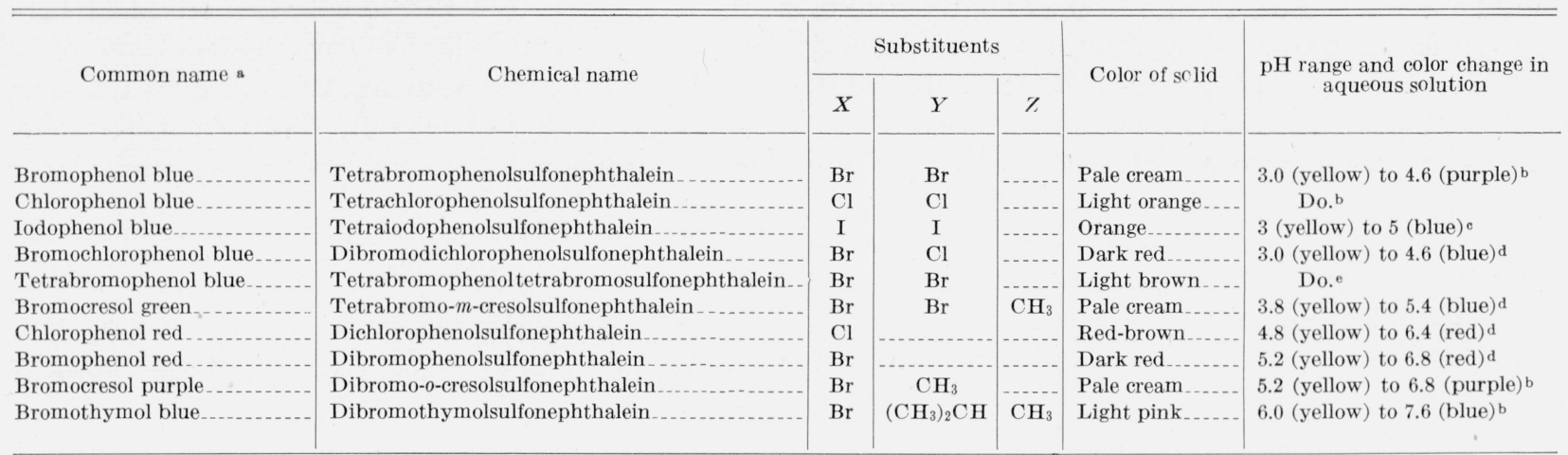

A The spelling used is that preferred by editors of publications of the American Chemical Society.

b H. A. Lubs and W. M. Clark, J. Washington Acad. Science 6, 481 (1916). The range 2.8 to 4.6 is given for bromophenol blue and for chlorophenol blue instead of the values 3.0 to 4.6 , now accepted.

- Eastman Organic Chemicals List No. 35, Eastman Kodak Co., Rochester, N. Y.

d B. Cohen, Reprint No. 1131 from the Public Health Reports (Government Printing Office, Washington, D. C., 1927).

- W. C. Harden and N. L. Drake, J. Am. Chem. Soc. 51, 562 (1929). M. M. Haring and H. A. Heller, J. Am. Chem. Soc. 63, 1024 (1941), give the pH range as 2.6 to 4.4 .

TABLE 2. Analyses of indicators

\begin{tabular}{|c|c|c|c|c|c|c|c|c|}
\hline \multirow{2}{*}{ Name } & \multicolumn{4}{|c|}{ Calculated } & \multicolumn{4}{|c|}{ Found a } \\
\hline & $\mathrm{C}$ & $\mathrm{H}$ & $X^{b}$ & $\mathrm{~S}$ & $\mathrm{C}$ & $\mathrm{H}$ & $X^{b}$ & $\mathrm{~S}$ \\
\hline & $\%$ & $\%$ & $\%$ & $\%$ & $\%$ & $\%$ & $\%$ & $\%$ \\
\hline Bromophenol blue c....... & 34.06 & 1. 50 & -...- & & 34.4 & 1. 7 & ...... & $\ldots$ \\
\hline Chlorophenol blue d... & 46. 37 & 2.05 & 28.82 & & 46.4 & 2.0 & 29.0 & \\
\hline Iodophenol blue d & 26.60 & 1. 18 & 59.17 & & 25.3 & 1.3 & 54.9 & - \\
\hline Tetrabromophenol blue d & 23.15 & 0.61 & 64.86 & & 28.9 & 1.4 & 57.8 & \\
\hline Bromocresol green ${ }^{c}$ & 36.13 & 2.02 & & & 36.4 & 2.8 & & \\
\hline Chlorophenol red: & & & & & & & & \\
\hline Sample $1{ }^{\circ} \ldots \ldots$ & 53.91 & 2.86 & 16. 75 & & 51.7 & 3.4 & & \\
\hline Sample $2 \mathrm{~d}$ & 53.91 & 2.86 & 16. 75 & & 52.4 & 3.0 & 18. 7 & .... \\
\hline Bromophenol red e........ & 44.55 & 2. 36 & 31.21 & 6. 26 & 44.4 & 2.8 & 31.2 & 6. 2 \\
\hline Bromocresol purple ${ }^{c}$ & 46. 70 & 2. 99 & $\ldots$ & $\ldots$ & 46.5 & 3. 2 & & $\ldots$ \\
\hline Bromothymol blue c & 51.94 & 4. 52 & & $\ldots$ & 52.0 & 4.5 & & -.... \\
\hline
\end{tabular}

a Average of two or three analyses. All samples were dried at low pressure before analysis.

b $X=\mathrm{Br}, \mathrm{Cl}$, or $\mathrm{I}$.

c Analysis was performed by Kenneth D. Fleischer.

d Analysis was performed by Rolf A. Paulson.

\section{Relation of Color to $\mathrm{pH}$ in Aqueous Media}

The reversible changes in color that phenol red, and other sulfonephthaleins in which alkyl or halogen substituents are present in the phenolic groups, undergo in aqueous solutions are the reason for their widespread use in the determination of $\mathrm{pH}$ values. The relation between the color and the $\mathrm{pH}$ of the solution can be understood by a consideration of the formulas shown in figure 2 . In aqueous media, three colored forms and a colorless form are known to exist:
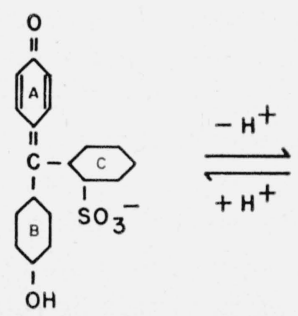

I

$$
-H^{+} \|+H^{+}
$$
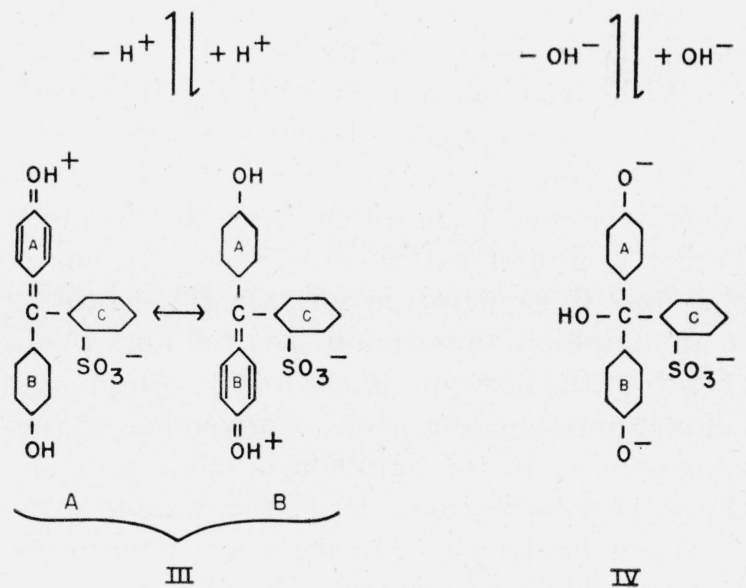

IV

Figure 2.-Structural and color changes of phenolsulfonephthalein (phenol red) in aqueous solutions.

I, Univalent anion (yellow); II, bivalent anion (deep color); III, amphion (deep color); IV, trivalent carbinol anion (colorless). 
Yellow form.-The yellow form is represented by formula I, namely, a univalent anion containing a quinoid group. This absorbs radiant energy in the violet region of the spectrum, and the color transmitted by its solutions is therefore yellow. The $\mathrm{pH}$ range over which this form is stable depends upon the substituents present. For phenolsulfonephthalein (phenol red) the range is roughly 2 to 6.5 , and for its tetrahalogenated derivatives the range is approximately -1 to 3 .

Deeply colored forms.- One deeply colored form is produced by the addition of a base to a solution of the yellow form. This change is brought about by the removal of phenolic protons, by combination with hydroxyl ions. The resulting bivalent anion (formula II, fig. 2) has a much deeper color than the univalent anion, because it absorbs light of longer wavelengths. The shift in absorption is attributed to resonance among various structures of nearly equivalent energy. The most important of these structures are most commonly believed to be those shown as $A$ and $B$ in formula II, figure 2 [5 to 11]. In the case of phenol red, the bivalent anion gives red solutions in water and is produced when the $\mathrm{pH}$ exceeds the approximate value 6.5. In the case of its tetrahalogen derivatives, the bivalent anion is purple or blue and is produced when the $\mathrm{pH}$ is greater than about 3.0.

A second deeply colored form is produced when a considerable quantity of acid is added to a solution of the yellow univalent anion. With phenol red, this change occurs in approximately $1-N$ hydrochloric acid, corresponding to a $\mathrm{pH}$ of about zero. With tetrahalogen derivatives, it occurs only when the indicator is dissolved in nearly concentrated hydrochloric or sulfuric acid. This second deeply colored form differs from the first one in having a proton attached to each oxygen in rings $A$ and $B$, as shown in formula III, figure 2 . It is an amphion (zwitterion, hybrid ion) $[5,6]$. The colors of the bivalent anions and the amphions are similar but not identical. Conversion of the univalent anion to the amphion is made possible by the weakly basic character of the quinoid oxygen. When halogen substituents are present in rings $A$ and $B$ in the positions ortho to oxygen, the basicity of the oxygen is lessened. This accounts for the difference in the concentrations of acid required for the conversion of halogenated and unhalogenated phenolsulfonephthaleins from the univalent anion to the amphion.
Colorless form. - The bivalent anion (formula II, fig. 2) is converted to the colorless trivalent carbinol anion (formula IV) by the addition of a hydroxyl ion to the central carbon. This reaction occurs at high $\mathrm{pH}$ values. The trivalent anion absorbs ultraviolet but not visible radiation.

\section{Color and Structure of Phenolsulfonephthaleins in the Solid State}

Of the indicators listed in table 1, the following have been isolated as colorless or nearly colorless solids: Bromophenol blue [12], bromochlorophenol blue [13], tetrabromophenol blue [14], bromocresol green [13, 15], bromocresol purple [16], and bromothymol blue [17]. Frequently, however, commercial specimens possess a pink tinge or even a dark red or brown color (see table 1, col. 4). Various impurities may be responsible in part for the color, but the most probable explanation is partial or complete hydration of the solid [12]. The colorless form of the solid is believed to have a lactone (sultone) structure (fig. 1). The deeply colored form of the solid is probably a hydrate of the amphion (formula III, fig. 2). The amphion structure is presumably stabilized by association of one or more water molecules with the $\mathrm{SO}_{3}$ group. This explanation is supported by the following facts: (1) Unhalogenated members of the phenolsulfonephthalein series, for example, phenol red and thymol blue, have been isolated only in hydrated, highly colored (dark red or chocolate-brown) forms $[13,15,17]$; as shown in the preceding section, these are the compounds that are most readily converted by the addition of acids into deeply colored amphions in aqueous media. (2) Halogenated members of the phenolsulfonephthalein series are obtainable in the colorless, anhydrous form from a nonaqueous medium, such as glacial acetic acid, but precipitate as deeply colored hydrates from aqueous solutions.

When the deeply colored solid is dissolved in water, the quinoid oxygen is no longer able to compete for the proton with the more basic as well as more numerous hydroxyl ions and water molecules. At one time the colorless lactone (sultone) form was believed to be present in aqueous solutions, in tautomeric equilibrium with the quinoid sulfonic acid corresponding to the univalent anion, formula I, figure 2 [5]. This view is not accepted at the present time [6]. 


\section{Reaction of Phenolsulfonephthaleins with Organic Bases in Benzene}

The phenolsulfonephthaleins investigated are all soluble to a limited extent in benzene. The lighter the color of the solid, the more readily the indicator dissolves. In fact, some deeply colored specimens are practically insoluble in benzene. These differences in solubility provide additional evidence that the deeply colored solid has an amphion structure. Solutions of the indicators in benzene are completely or nearly colorless. The solid apparently dissolves as the lactone (fig. 1). In most instances the solution becomes a pale yellow color on standing, and a noticeable yellow stain appears on the glass of the container, particularly around the neck and the stopper. The yellow color of the solution is most evident on days of high humidity and is probably partly caused by moisture adsorbed by the sample or by the glass. It should also be remembered that many glass vessels show an alkaline reaction, because of the chemical composition of the glass or localized deposits of alkali on the surface. ${ }^{2}$ The yellow color indicates partial conversion to the quinoid structure.

Either strong or weak bases, such as aliphatic amines or aniline derivatives, cause the appearance or intensification of the yellow color when added in minute amounts to a benzene or chlorobenzene solution of an indicator of this group, for example, bromophenol blue $[18,19]$. A further change in color is brought about when more than a molar equivalent of a strong base is added to the solution. In the work of previous investigators, apparently the only organic bases employed were secondary aliphatic amines, which produced a blue color, as was to be expected by analogy with aqueous solutions $[18,19] .^{3} \quad$ We discovered that the color produced in inert solvents such as benzene and chlorobenzene depends on the chemical type to which the base belongs. In short the reactions of organic bases of various

\footnotetext{
${ }^{2}$ Elizabeth E. Murray, formerly of this Bureau, observed that several new volumetric flasks, said to have been cleaned with an acid mixture, acquired blue spots on the inner surface after being filled with a benzene solution of bromophenol blue. The blue spots no longer formed after the flasks had been kept filled with dilute hydrochloric acid for several days and then thoroughly washed and dried.

${ }^{3}$ Bronsted appears to be the first person to have used indicators of the sulfonephthalein series for the study of acid-base reactions in benzene. Piperidine, benzylamine, and isoamylamine were the bases used in his exploratory investigations. Experimental details were not given, and the color changes observed were not described [20].
}

classes with phenolsulfonephthaleins and related indicators in benzene and other inert or "aprotic" solvents do not exactly parallel those observed in aqueous media but reveal differences that are masked in an "amphiprotic" solvent, such as water. In the first paper of this series, the reactions of bromophthalein magenta with various organic bases were described at length, and analogous reactions of phenolsulfonephthaleins were referred to [1]. Experimental observations and data for sulfonephthaleins will now be presented.

\section{Data and Observations}

\section{Transmittancy Curves for Phenolsulfone- phthaleins in Benzene}

In figure 3 are presented transmittancy curves for three halogen derivatives of phenolsulfonephthalein and, for comparison, the curves for two related derivatives of phenolphthalein. The intended concentration in all cases was $5 \times 10^{-5} \mathrm{M}$ but, as already explained, the sulfonephthaleins react with moisture or a glass surface, the iodophenol blue appeared to be impure, and it is not certain that the tetrabromophenolphthalein was free of impurities. However, the solutions can

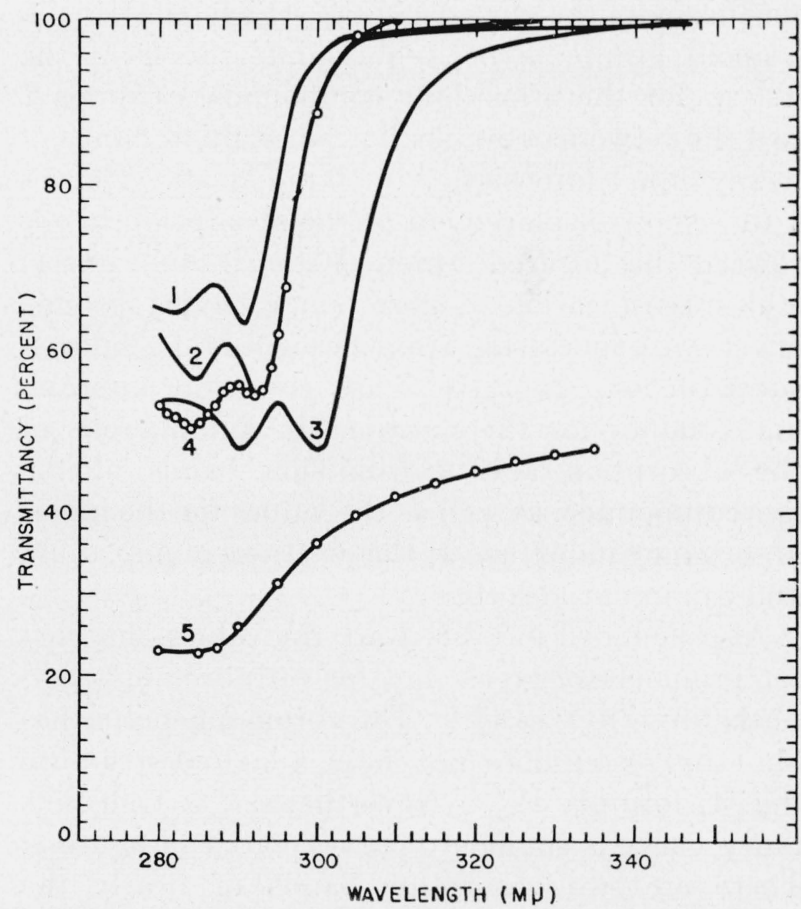

Figure 3. Transmittancy curves for sulfonephthaleins and related indicators in benzene, all approximately $5 \times 10^{-5} \mathrm{M}$.

(1) Chlorophenol blue; (2) bromophenol blue; (3) iodophenol blue; (4) tetrabromophenolphthalein; (5) bromophthalein magenta $E$. 


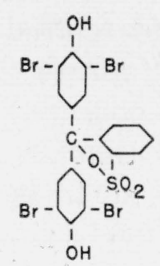

I

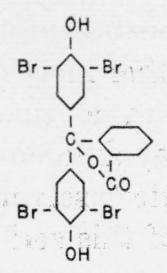

II

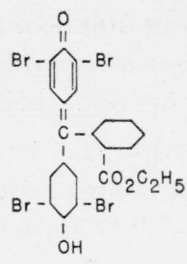

III

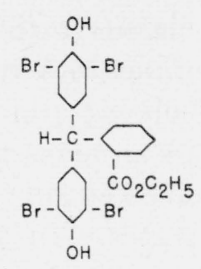

IV
Figure 4. Structural formulas for the modifications existing in benzene solution.

I, Bromophenol blue; II, tetrabromophenolphthalein; III, bromophthalein magenta $E$ (tetrabromophenolphthalein ethyl ester); IV, tetrabromophenolphthalin ethyl ester.

be considered as very nearly equivalent in concentration. Curves 1 to 4 in figure 3 are for chlorophenol blue, bromophenol blue, iodophenol blue, and tetrabromophenolphthalein, respectively. Curve 5 is for bromophthalein magenta (tetrabromophenolphthalein ethyl ester). In figure 4 are shown the structures ascribed to the modifications of bromophenol blue, tetrabromophenolphthalein, and bromophthalein magenta that exist in an inert solvent such as benzene (formulas I to III, respectively). Chlorophenol blue and iodophenol blue have, of course, the same structural pattern as bromophenol blue. The curve for bromophthalein magenta, which contains a quinoid group, is noticeably different from the curves for the other four compounds. Curves 1 to 4 show two narrow, shallow absorption bands of nearly equal intensity, 7 to $10 \mathrm{~m} \mu$ apart. Curves 1 to 3 show displacement of the absorption bands toward the infrared region (bathochromic effect) and intensification of absorbancy (hyperchromic effect) with increasing atomic weight of the halogen substituents. In table 3 are given the approximate values for the positions in millimicrons of the absorption and transmission bands of the five compounds, as well as the values for the molar absorbancy index, $a_{M}$, at the positions of maximum and minimum absorbancy.

Also included in table 3 are the values obtained for bromocresol green and for tetrabromophenolphthalin ethyl ester. ${ }^{4}$ Tetrabromophenolphthalin ethyl ester does not have a lactoid structure (fig. 4, formula IV). Nevertheless, its transmittancy curve is similar to curves 1 to 4 in figure 3 . There are two absorption bands of nearly the same intensity, $8 \mathrm{~m} \mu$ apart.

\footnotetext{
${ }^{4}$ Spectrophotometric data for benzene solutions of tetrabromophenolphthalin ethyl ester and chlorophenol blue were obtained by E. Anne McDonald of this Bureau.
}

TABLE 3. Approximate values for molar absorbancy indexes and positions (in millimicrons) of absorption and transmission bands of benzene solutions

\begin{tabular}{|c|c|c|c|c|c|c|}
\hline \multirow{2}{*}{ Compound } & \multicolumn{2}{|c|}{$\begin{array}{l}\text { First absorp- } \\
\text { tion band }\end{array}$} & \multicolumn{2}{|c|}{$\begin{array}{l}\text { Second absorp- } \\
\text { tion band }\end{array}$} & \multicolumn{2}{|c|}{$\begin{array}{l}\text { Transmission } \\
\text { band }\end{array}$} \\
\hline & $\begin{array}{l}\text { Posi- } \\
\text { tion }\end{array}$ & $a_{M}$ & $\begin{array}{l}\text { Posi- } \\
\text { tion }\end{array}$ & $a_{M}$ & $\begin{array}{l}\text { Posi- } \\
\text { tion }\end{array}$ & $a_{M}$ \\
\hline Chlorophenol blue...... & 282 & 3,950 & 290.5 & 4,000 & 287 & 3,300 \\
\hline Bromophenol blue & 285 & 5,000 & 292.5 & 5,250 & 288.5 & 4,300 \\
\hline Iodophenol blue ........ & 290 & 6,450 & 300 & 6,400 & 295 & 5,400 \\
\hline $\begin{array}{l}\text { Tetrabro mophenol- } \\
\text { phthalein }\end{array}$ & 284 & 5,950 & 291.5 & 5,300 & 289.5 & 5,150 \\
\hline Bromophthalein magenta & 285 & 13,000 & & & & ...... \\
\hline $\begin{array}{l}\text { Tetrabro mophenol- } \\
\text { phthalin ethyl ester... }\end{array}$ & 286 & 7,700 & 294 & 7,450 & 291 & 7,200 \\
\hline Bromocresol green...... & 284 & 3,850 & 292.5 & 4,000 & 288.5 & 3,600 \\
\hline
\end{tabular}

\section{Bromocresol Green}

Transmittancy curves for approximately $5 \times 10^{-5}-M$ bromocresol green in benzene, with and without added acid or base, are shown in figures 5 to 7.

The curves shown in figure 5 were obtained for mixtures of bromocresol green in benzene with the following: $(A)$ Solid line, $2.5 \times 10^{-4}-M$ acetic acid

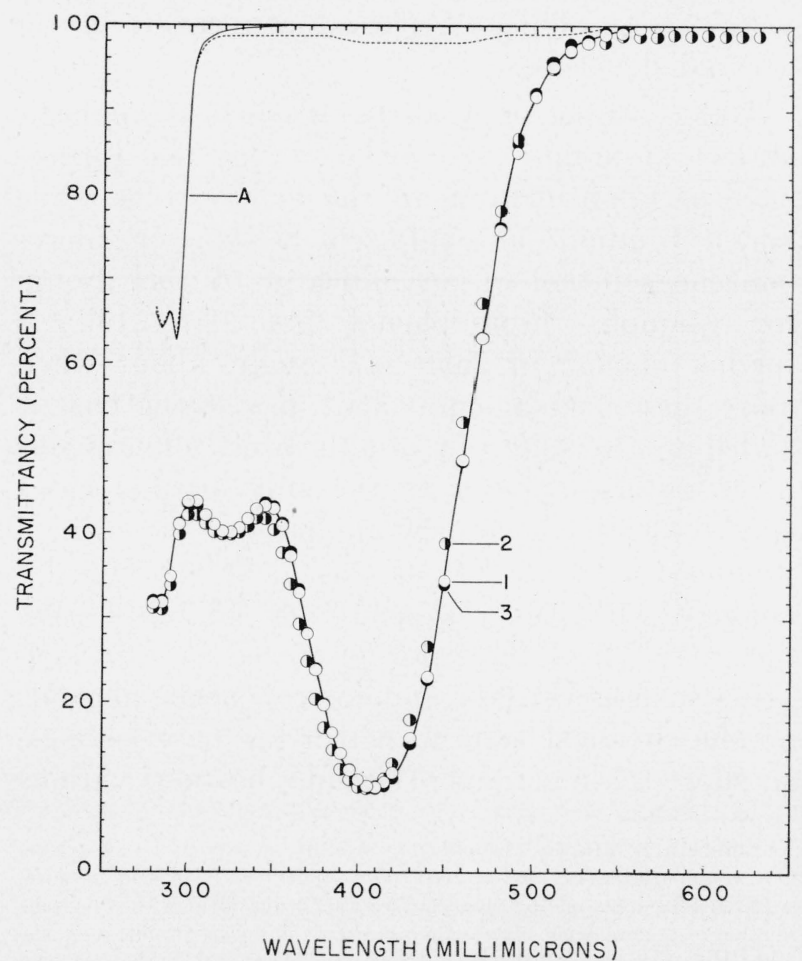

FIgure 5. Transmittancy curves for $5 \times 10^{-5}-\mathrm{M}$ bromocresol green in benzene.

(A) solid line, plus 5 molar equivalents of acetic acid; (A) dotted line, without acid. (1) to (3), plus 1 molar equivalent of mono- $n$-amylamine, di- $n$ amylamine, and tri- $n$-amylamine, respectively. 


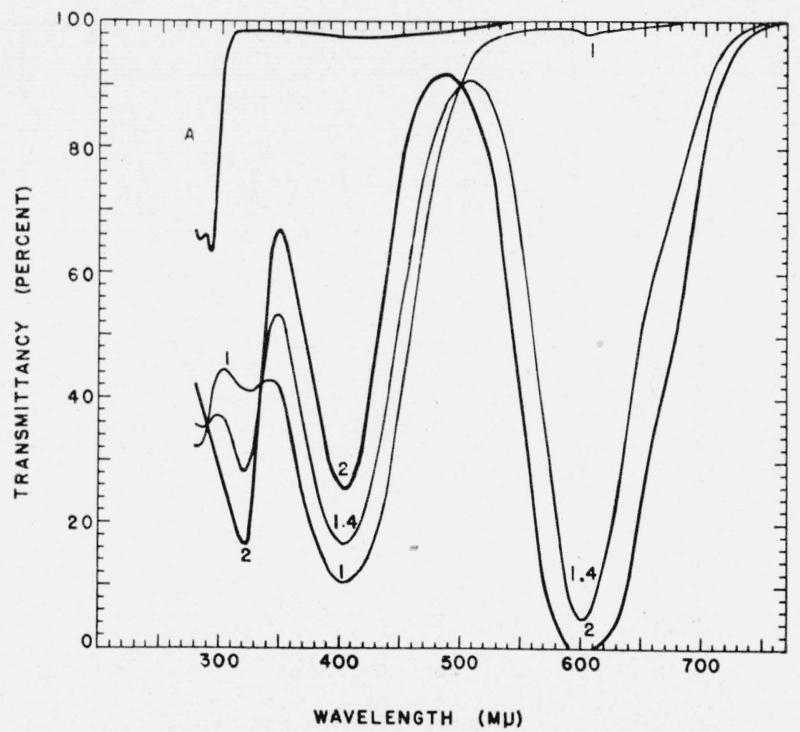

FiguRE 6. Transmittancy curves for $5 \times 10^{-5}-M$ bromocresol green in benzene and for its mixtures with piperidine.

(A), Without piperidine; (1), (1.4), (2), mixtures with 1, 1.4, and 2 molar equivalents of piperidine.

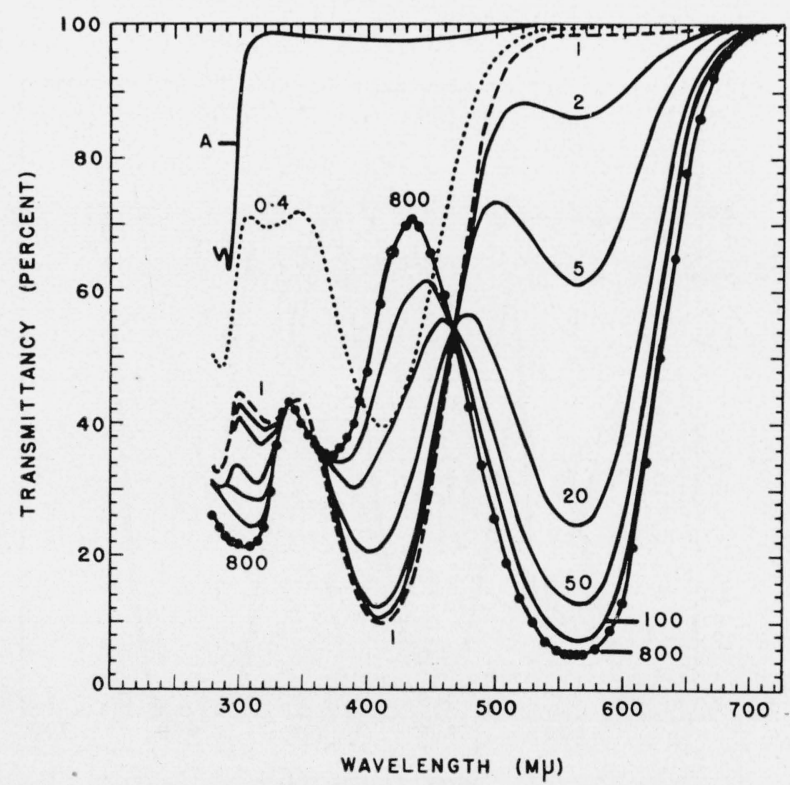

Figure 7. Transmittancy curves for $5 \times 10^{-5}-M$ bromocresol green in benzene and for its mixtures with triethylamine.

(A), Without triethylamine; (0.4) to (800), mixtures with from 0.4 to 800 . molar equivalents of triethylamine.

(5 molar equivalents $)^{5} ;(A)$ dotted line, no acid; (1) $5 \times 10^{-5}-M$ mono- $n$-amylamine ( 1 molar equivalent); (2) 1 molar equivalent of di-n-amylamine; (3) 1 molar equivalent of tri- $n$-amylamine. The two curves marked $A$ show that bromocresol green

\footnotetext{
${ }_{5}^{5}$ The comparison cell contained $2.5 \times 10^{-4}-M$ acetic acid in benzene.
}

in benzene was partly converted into the yellow quinoid form, and that with a moderate excess of glacial acetic acid these traces of the quinoid form were converted into the lactone. The effect of the acetic acid is probably to be attributed to its combination with traces of water in the solution. Mono-, di-, and tri-n-amylamine produce almost identically the same change in the absorption curve when their molar concentration is the same as that of the indicator. Curves 1 to 3 show absorption bands near 285 and $410 \mathrm{~m} \mu$, and transmission bands near 300 and $345 \mathrm{~m} \mu$. Approximate values for $a_{M}$ found for the four bands, in the sequence given, are $10,000,20,000,7,200$, and 7,200 .

The transmittancy curves in figure 6 show the changes in the absorbancy of $5 \times 10^{-5}-M$ bromocresol green in benzene that occurred when more than 1 molar equivalent of piperidine was added. The curves are for the indicator alone and for solutions that contained $1,1.4$, and 2 molar equivalents of piperidine as well. The original faintly yellow solution became a vivid yellow after the addition of 1 molar equivalent of the base. When the quantity of base was increased by only 0.4 molar equivalent, the color changed to blue. From figure 6 it can be seen that the appearance of a strong absorption band near $600 \mathrm{~m} \mu$ and a relatively small decrease in the absorbancy near $400 \mathrm{~m} \mu$ accompanied this change in color. Curve 1 in figure 6 and curve 2 in figure 5 show the beginning of a change in the same direction. Curves 1, 1.4, and 2 show well-marked isosbestic points near 335 and 495 to $500 \mathrm{~m} \mu$. Di-n-amylamine, when added in excess of 1 molar equivalent, produced the same change as piperidine. The quantity of secondary amine required for complete conversion of bromocresol green to the blue form was not determined.

Curves 0.4 to 800 in figure 7 show the changes that occurred after the addition of $0.4,1,2,5,20$, 50,100 , and 800 molar equivalents of triethylamine to $5 \times 10^{-5}-M$ bromocresol green in benzene. The reaction appeared to be complete after 800 molar equivalents or less of triethylamine had been added. Comparison of the transmittancy curves presented in figure 7 with those in figure 6 reveals that the addition of 1 molar equivalent of triethylamine produced essentially the same effect as the addition of a molar equivalent of piperidine or mono-, di-, or tri- $n$-amylamine. When the con- 
centration of triethylamine became greater than a molar equivalent, the color changed toward magenta instead of to the blue produced by the addition of piperidine or diamylamine. It is obvious that curves 2 to 800 in figure 7 are very different from curves 1.4 and 2 in figure 6 . The magenta solutions show a broad absorption band near $565 \mathrm{~m} \mu$ (instead of a narrower, more intense band near $600 \mathrm{~m} \mu$ ) and the isosbestic points occur near 360 and 465 to $470 \mathrm{~m} \mu$ (instead of near 335 and 495 to $500 \mathrm{~m} \mu$ ). 1,2-Diphenylguanidine caused the same change as triethylamine-a change first to yellow and then to magenta with intermediate orange and red tones. After standing, a precipitate began to form in some of the solutions. For example, a solution to which 2 molar equivalents of diphenylguanidine had been added showed a Tyndall beam after standing overnight, but a solution that contained only 1 molar equivalent of diphenylguanidine was still clear. With di-o-tolylguanidine, a precipitate was evident when more than about 1.6 molar equivalents of the base had been added.

\section{Bromophenol Blue}

The behavior of bromophenol blue in benzene with typical secondary and tertiary aliphatic amines is very similar to that of bromocresol green. Transmittancy curves for approximately $5 \times 10^{-5}-M$ bromophenol blue in benzene, with and without added base, are shown in figures 8 to 11 .

The effects of $0.2,0.4,0.6,0.8$, and 1.0 molar equivalent of di-n-butylamine are shown in figure 8. Curve $A$ is for bromophenol blue without added acid or base; here again, the presence of a small amount of the yellow quinoid form is evident. Curves 0.2 to 0.8 show a gradual intensification of the absorption band near $400 \mathrm{~m} \mu$, characteristic of the quinoid form. It is interesting to note that the absorption band near $292.5 \mathrm{~m} \mu$ gradually disappears as the band near $400 \mathrm{~m} \mu$ becomes stronger. When the concentration of dibutylamine is increased from 0.8 to 1.0 molar equivalent, the absorbancy near $400 \mathrm{~m} \mu$ begins to diminish, and a very strong absorption band near $575 \mathrm{~m} \mu$ becomes evident. Evidently the second stage in the neutralization of bromophenol blue begins before the first stage is complete.

The transmittancy curves in figure 9 show the changes that occur when the concentration of di-

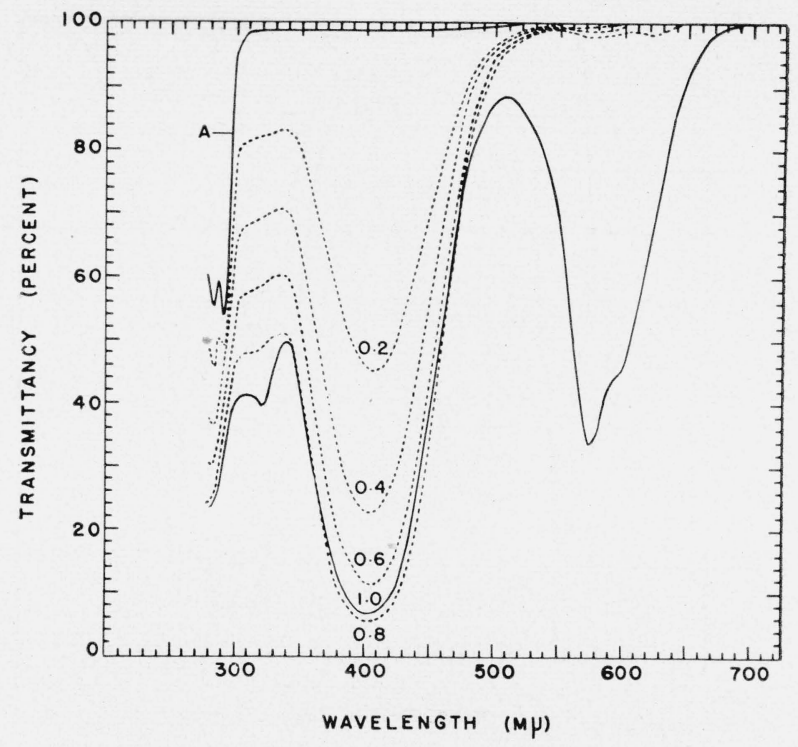

Figure 8. Transmittancy curves for $5 \times 10^{-5}-M$ bromophenol blue in benzene and for its mixtures with di-nbutylamine.

(A), Without dibutylamine; (0.2) to (1.0), mixtures with from 0.2 to 1.0 molar equivalents of dibutylamine.

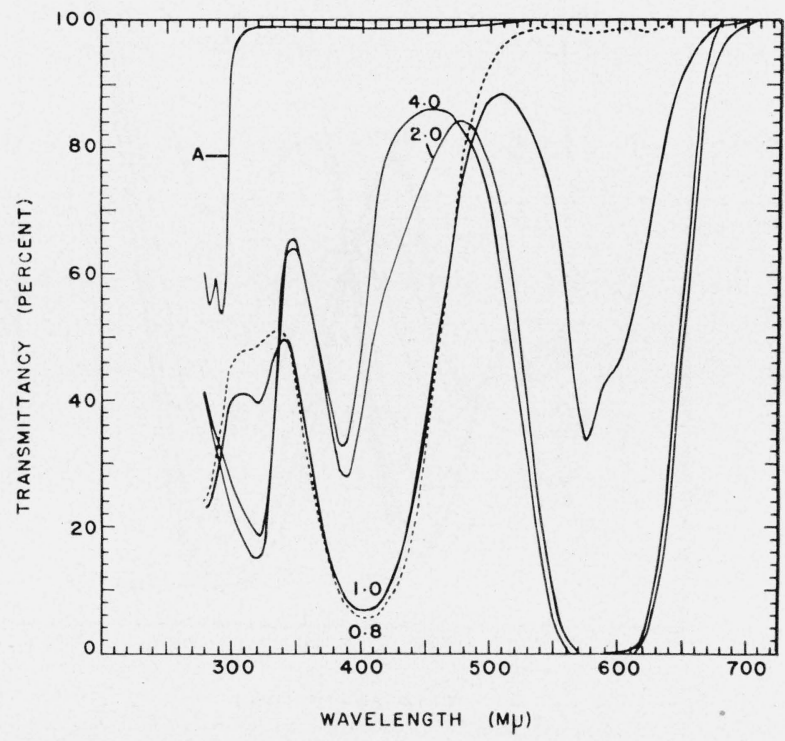

Figure 9. Transmittancy curves for $5 \times 10^{-5}-M$ bromophenol blue in benzene and for its mixtures with di-nbutylamine.

(A), Without dibutylamine; (0.8) to (4.0), with from 0.8 to 4.0 molar equivalents of dibutylamine.

$n$-butylamine is increased successively from 0.8 molar equivalent to $1.0,2.0$, and 4.0 molar equivalents. The solutions were green to blue in color. When figures 6 and 9 are compared, it is seen that the changes produced by the addition of a second- 


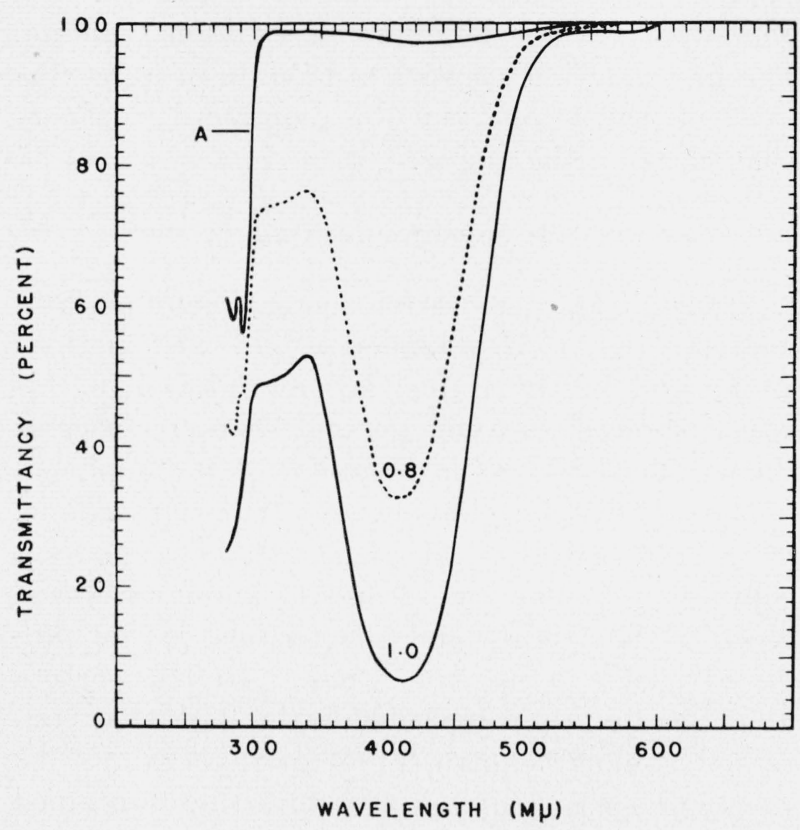

Figure 10. Transmittancy curves for $5 \times 10^{-5}-M$ bromophenol blue in benzene and for its mixtures with tri-nbutylamine.

(A), Without tributylamine; (0.8) and (1.0), with 0.8 and 1.0 molar equivalent of tributylamine, respectively.

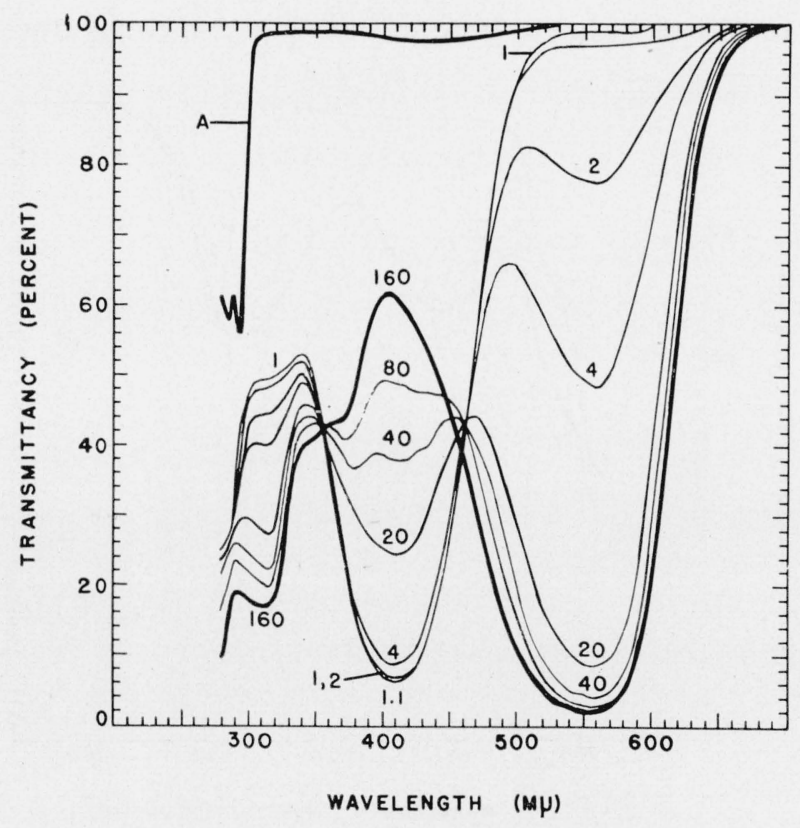

Figure 11. Transmittancy curves for 5x10-5-M bromophenol blue in benzene and for its mixtures with tri-nbutylamine.

(A), Without tributylamine; (1) to (160), with from 1 to 160 molar equivalents of tributylamine. ary amine to bromocresol green and to bromophenol blue are of the same general character. No attempt was made to complete the spectrophotometric study of the reaction between bromophenol blue and dibutylamine because of the absence of sharply defined isosbestic points and changes that occurred in the transmittancy curves after the solution had stood for a short time.

The transmittancy curves in figure 10 show the effect of adding 0.8 and 1.0 molar equivalent of tri- $n$-butylamine to bromophenol blue. The curves marked 0.8 and 1.0 conform to the pattern of the curves in figure 8 . However, tributylamine appears to be less reactive than dibutylamine, as the change caused by the addition of 0.8 molar equivalent of tributylamine was intermediate between the effects of 0.2 and 0.4 molar equivalent of dibutylamine. The relative strengths of dibutylamine and tributylamine in water, as measured by their ionization constants, are the same as in benzene (compare p. 235 and 238, reference [1]).

The transmittancy curves in figure 11 show the changes that are produced by the addition of more than 1 molar equivalent of tri- $n$-butylamine to bromophenol blue. Curves are given for the reaction of the indicator with $1,1.1,2,4,20,40$, 80 , and 160 molar equivalents of tributylamine. The colors of the solutions were yellow to magenta, with intermediate orange and red tones. The main absorption band for the magenta solution is near $555 \mathrm{~m} \mu$, and the isosbestic points are near 355 and $460 \mathrm{~m} \mu$. The curves are of a different pattern from those shown in figure 9 for the reaction of bromophenol blue with dibutylamine but resemble the transmittancy curves for the reaction of bromocresol green with triethylamine (see fig. 7). The solution of bromophenol blue that contained 160 molar equivalents of tributylamine changed after standing, and the curve does not pass through the isosbestic point near $460 \mathrm{~m} \mu$. Although quantitative comparisons were not possible because of the instability of the solutions, bromophenol blue appeared to be more reactive than bromocresol green with amines in benzene. This difference is in harmony with the relative acidic strengths of the two indicators in water (see table 1, col. 5).

Reaction of bromophenol blue with primary amines.-One molar equivalent of $n$-amyl- or $n$-heptylamine produced a vivid yellow color 
when added to $5 \times 10^{-5}-M$ bromophenol blue. The addition of a larger amount of the primary amine caused a momentary vivid color, but a precipitate formed almost at once. With slightly more than a molar equivalent of 0 -aminodicyclohexyl, there was evidence of an absorption band near $575 \mathrm{~m} \mu$.

Quantitative aspects.-Because of the tendency of benzene solutions of bromophenol blue to acquire a pale yellow tint and to produce a yellow stain on the glass, measurements of high precision are not readily performed with this indicator. However, it is possible to follow semiquantitatively its reactions with secondary and tertiary amines in benzene. Examples of such measurements are presented in figure 12. The absorbancy

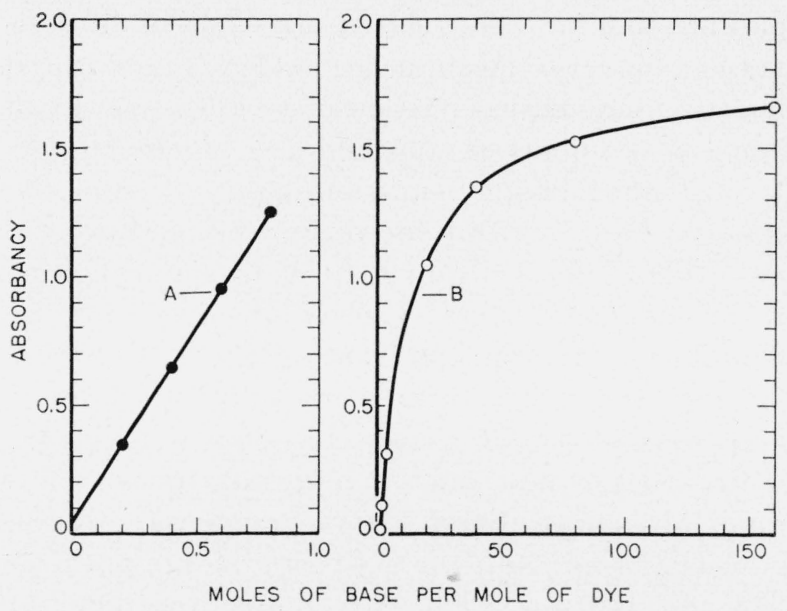

FIGURE 12. Change in absorbancy produced by adding different amounts of base, expressed in molar equivalents of the indicator, to approximately $5 \times 10^{-5}-M$ bromophenol blue in benzene.

(A) Bromophenol blue plus di- $n$-butylamine, $405 \mathrm{~m} \mu$; (B) Bromophenol blue plus tri- $n$-butylamine, $550 \mathrm{~m} \mu$.

(optical density) at a fixed wavelength was plotted against the number of moles of base per mole of the indicator dye. The experimental points in curve $A$ were obtained by measuring the absorbancy at $405 \mathrm{~m} \mu$ of mixtures of approximately $5 \times 10^{-5}-M$ bromophenol blue with $0.2,0.4,0.6$, and 0.8 molar equivalent of di- $n$-butylamine; the reaction involved was the conversion of the colorless lactone to the yellow acid salt. The experimental points in curve $B$ are for the conversion of the yellow acid salt to the magenta form; measurements were made at $550 \mathrm{~m} \mu$ with solutions that contained approximately $5 \times 10^{-5}-M$ bromophenol blue with various amounts of tri- $n$-butylamine. The first reaction appears to have been quantitative, but the second reaction required a considerable excess of the amine.

\section{Iodophenol Blue}

Transmittancy curves for the reaction of approximately $5 \times 10^{-5}-M$ iodophenol blue with diethylamine and triethylamine, not reproduced in this paper, showed the same general characteristics as those described for the reaction of bromophenol blue with di- $n$-butylamine and tri- $n$-butylamine. With both indicators, after the initial change from colorless to yellow, the secondary amine produced green to blue tones and the tertiary amine produced orange to magenta tones. After a week, a green precipitate was visible in the flask that contained a molar equivalent of diethylamine. No precipitate was visible in the flask that contained 4 molar equivalents of triethylamine, and the solution did not show a Tyndall beam.

\section{Tetrabromophenol Blue}

In figure 13 are shown representative transmittancy curves for approximately $2.5 \times 10^{-5}-M$ tetrabromophenol blue in benzene (curve $A$ ) and

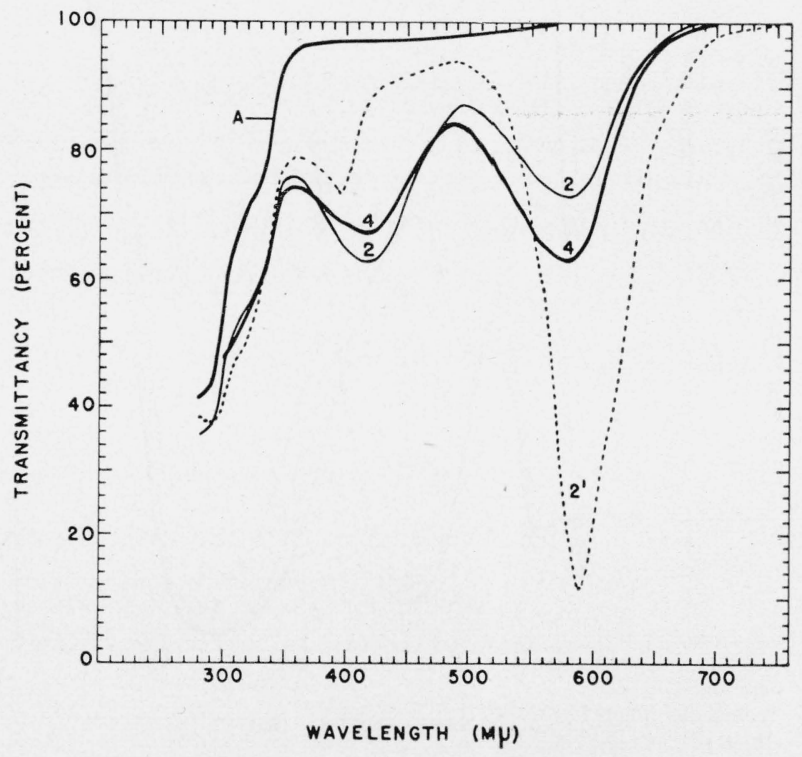

Figure 13. Transmittancy curves for $2.5 \times 10^{-5}-M$ tetrabromophenol blue in benzene and for its mixtures with triethylamine and diethylamine.

(A) Without base; (2) with 2 molar equivalents of triethylamıe; (4) with 4 molar equivalents of triethylamine; $\left(2^{\prime}\right)$ with 2 molar equivalents of diethylamine. 
for its mixtures with 2 or 4 molar equivalents of triethylamine and with 2 molar equivalents of diethylamine (curves 2, 4, and 2', respectively). The transmittancy curve for tetrabromophenol blue, which has 4 bromine atoms in ring $C$ (see fig. 1), is unlike the transmittancy curves previously shown for phenolsulfonephthalein derivatives that have no halogen in ring $C$ (see figs. 3 and 5). Tetrabromophenol blue is also more difficultly soluble in benzene than the other phenolsulfonephthaleins discussed in this paper. However, the change in absorbancy caused by the addition of 2 to 4 molar equivalents of triethylamine is of a distinctly different character from the change produced by 2 molar equivalents of diethylamine. In other words, tetrabromophenol blue resembles bromocresol green and bromophenol blue in reacting differently with secondary and tertiary aliphatic amines. The reaction of $2.5 \times 10^{-5}-M$ tetrabromophenol blue with triethylamine in benzene appeared to be complete when about 150 molar equivalents of the base had been added.

\section{Other Halogenated Phenolsulfonephthaleins}

Qualitative observations of the behavior of six other halogenated phenolsulfonephthaleins are summarized in table 4. Solutions of the indicators used for the tests were prepared by warming the solid with benzene, allowing the solution to cool to room temperature, and then filtering it from any undissolved solid. The following conclusions can be made: 1 . The initial change, from colorless or pale yellow to deep yellow, is the same for all the indicators, irrespective of the type of amine added. 2. The second change, from yellow to a deeper color, produced by the addition of piperidine, is of the same character for all the indicators. 3. The change from yellow to a deeper color, produced by the addition of triethylamine, is similar for all the indicators but different from the effect of piperidine. 4. With all the indicators except bromothymol blue, an excess of $n$-butylamine causes the formation of a precipitate. 5. Bromothymol blue is less reactive with amines in benzene than are the other indicators listed in table 4 , just as it is less sensitive to alkali in aqueous media. 6. The first five indicators listed in table 4 show qualitatively, at least, the same behavior as bromocresol green, bromophenol blue, iodophenol blue, and tetrabromophenol blue.

Phenol red and the unhalogenated derivatives of phenol red that are available commercially were found to be practically insoluble in benzene. Even if soluble, they would not be expected to possess sufficiently great acidity to be useful indicators for inert media.

\section{Discussion}

\section{Comparison of Bromophenol Blue with Bromophthalein Magenta}

It has been shown in section $\mathrm{V}$ that halogenated derivatives of phenolsulfonephthalein react differently in benzene with primary, secondary, and tertiary alphatic amines. Bromophthalein magenta also shows a differentiating behavior with amines of different classes, and the explanation previously given for this [1] can be applied to the sulfonephthaleins. The nearest sulfonephthalein analog of bromophthalein magenta $E$ is bromophenol blue. A comparison of the two indicators reveals interesting similarities and differences.

TABLE 4.-Effect of base upon the color of halogen derivatives of phenolsulfonephthalein in benzene

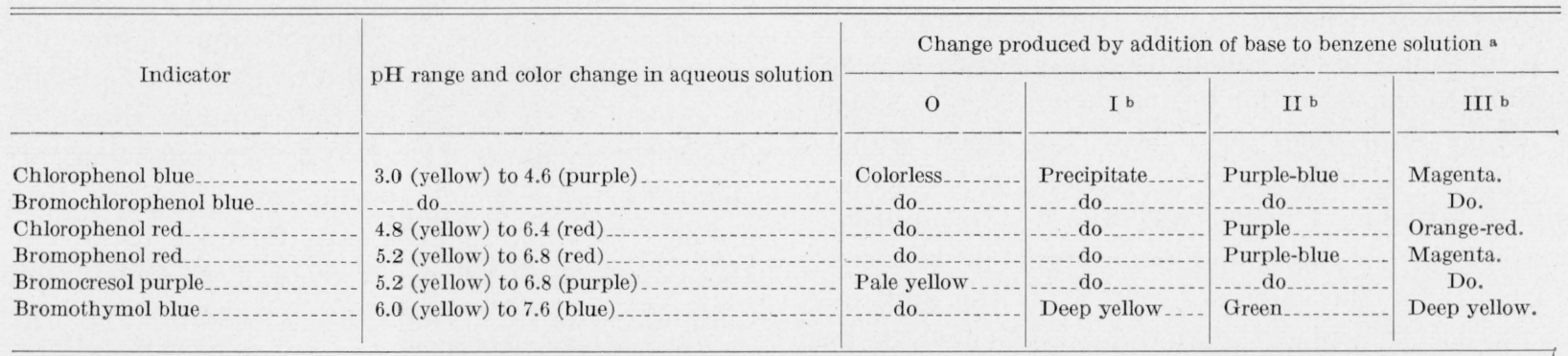

\footnotetext{
a Key to bases used: $\mathrm{O}=$ no base; $\mathrm{I}=n$-butylamine; $\mathrm{II}=$ piperidine; $\mathrm{III}=$ triethylamine.

b If the amine was added gradually, in all cases the first change was the appearance or the intensification of a yellow color. The color given in the table is the one observed after the addition of a moderate excess of the amine.
} 
Bromophenol blue is nearly colorless in the solid state and in benzene or other inert solvents, ${ }^{6}$ whereas bromophthalein magenta is brick-red in the solid state and gives yellow solutions. These differences are explicable if the lactoid structure (formula I, fig. 4) is assigned to bromophenol blue; if the indicator existed as the isomeric quinoid sulfonic acid, it would give a yellow solution in benzene and would be yellow, orange, or red in the solid state. The only structure assignable to bromophthalein magenta, that of a quinoid carboxylic ester (formula III, fig. 4), is in accord with the deep color of the solid and the yellow color of its solution in benzene.

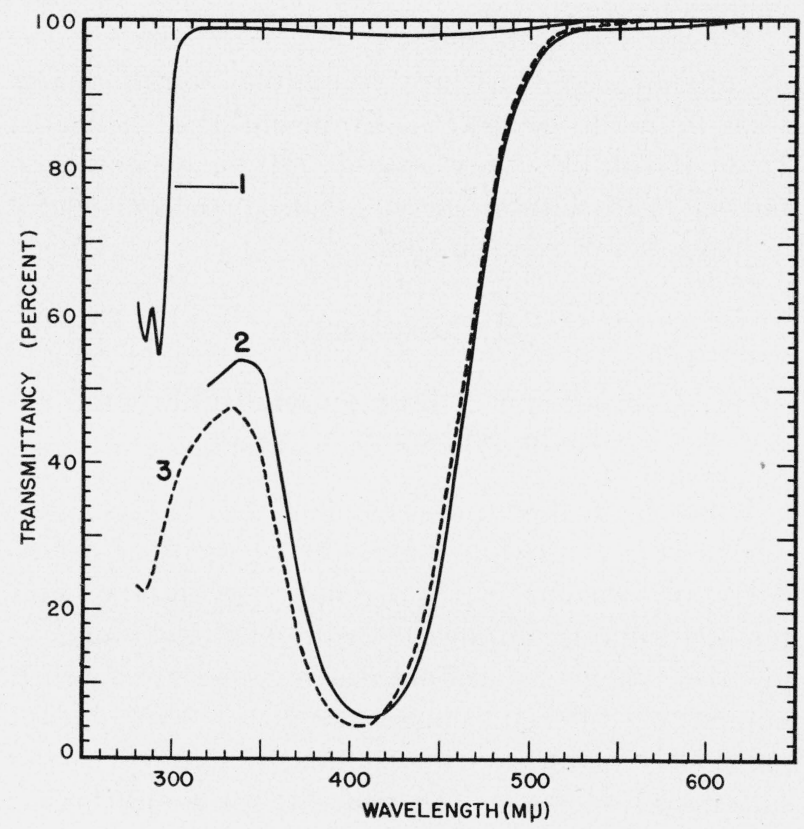

Figure 14. Transmittancy curves for bromophenol blue and bromophthalein magenta $E, 5 \times 10^{-5}-M$ in benzene.

(1) Bromophenol blue; (2) bromophenol blue plus 1 molar equivalent of 1,2-diphenylguanidine; (3) bromophthalein magenta $E$.

As shown in figure 14, the transmittancy curve for bromophthalein magenta in benzene is very similar to the curve for bromophenol blue to which 1 molar equivalent of a base has been added. Both curves show a strong absorption band near 405 to $410 \mathrm{~m} \mu$. Curve 1 was obtained for approximately $5 \times 10^{-5}-M$ bromophenol blue in benzene; curve 2 , for the same solution after the addition of $5 \times 10^{-5}-M$ diphenylguanidine; and curve 3 , for $5 \times 10^{-5}-M$ bromophthalein magenta $E$ in benzene.

6 See table 1 , col. 4 , and the discussion on p. 31 .
The solution that gave curve 1 was nearly colorless, and the solutions that gave curves 2 and 3 were both a vivid yellow.

It should be noted, however, that bromophthalein magenta $E$ is a monobasic acid, but that bromophenol blue in its yellow solutions in benzene is an acid salt. The mechanism of the conversion of bromophenol blue to the acid salt is puzzling. The explanation along classical lines would have assumed the following two steps: (1) A spontaneous change of the lactoid form of the sulfonephthalein in benzene into the tautomeric quinoid sulfonic acid until equilibrium between the two forms was established; (2) the reaction of any added base with the quinoid sulfonic acid to give a salt, with a consequent displacement of the equilibrium between the lactone and the quinoid sulfonic acid and eventual complete conversion of the lactone into the salt of the quinoid sulfonic acid. Such a mechanism requires either the spontaneous wandering of a proton from one part of the molecule to a relatively remote portion, or else the alinement of two molecules in a suitable position for the transfer of protons from - $\mathrm{OH}$ groups to the $-\mathrm{SO}_{2} \mathrm{O}$ groups. The first explanation is unsatisfactory, for it is now believed that the probability of the release of a proton is practically negligible unless a second atom is present to which it is more strongly attracted. The second explanation seems improbable, because the proton is less strongly attached to the sulfonate group than to a phenolic oxygen. Although there is in most cases a yellow tinge to the solutions of the sulfonephthaleins in benzene, as pointed out on p. 31, the authors believe that this is due to traces of moisture or to contact with an alkaline surface such as glass, instead of to the spontaneous change of the lactone to the quinoid sulfonic acid.

A third possible mechanism for the conversion of the colorless lactone to the yellow acid salt in benzene is as follows: (1) The proton of a phenolic group forms a bridge to the nitrogen of a molecule of an amine, $\mathrm{R}_{3} \mathrm{~N}$, giving the complex shown in figure 15, formula II; (2) a redistribution of electrons takes place within the complex, producing the quinoid acid salt, formula III. The change of group $A$ or $B$ from a phenolic to a quinoid structure may be attributed to the combined effect of the attraction toward nitrogen of the phenolic proton and the attraction toward oxygen of the electron pair that originally united 


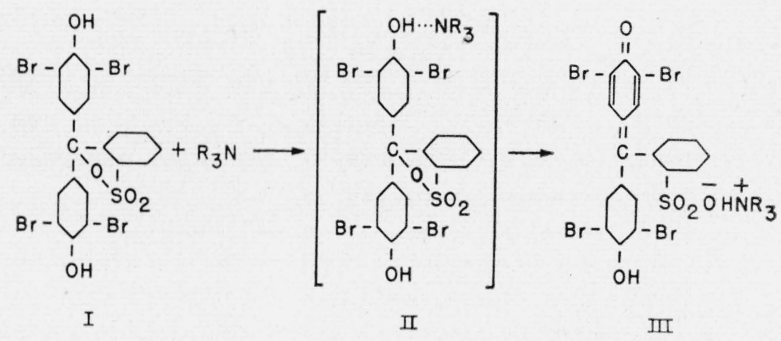

FIgure 15. Suggested mechanism for the conversion of bromophenol blue to its acid salt in benzene.

I, Bromophenol blue; II, intermediate complex; III, acid salt.

the methane carbon and the sulfonate group. ${ }^{7}$ The colorless lactoid form of the phenolsulfonephthaleins can exist only in inert solvents. Water is sufficiently basic to convert the lactone into the quinoid sulfonic acid, and the lactone is therefore not detected in aqueous solutions.

The changes in color that accompany the second step in the neutralization of bromophenol blue and other halogenated phenolsulfonephthaleins in benzene resemble, in general, the changes that occur when organic bases are added to a benzene solution of bromophthalein magenta. These changes may be summarized as follows: Primary aliphatic amines produce a red-purple color; secondary aliphatic amines, a purple-blue color; tertiary aliphatic amines or symmetrical di- or triarylguanidines, a magenta color; and the quaternary ammonium salts give blue solutions without a purple tinge. The chief difference that we observed between the salts of the sulfonephthaleins and those of bromophthalein magenta is the much lower solubility of the former. This is particularly true of the products formed by the reaction of the indicators with primary aliphatic amines.

From a systematic spectrophotometric investigation of the differently colored products from the reaction of bromophthalein magenta with amines of various classes and observations of the effect of the nature of the solvent, it was concluded that the magenta trialkylammonium salt has the monomeric structure shown in figure 16, formula I, and the purple-blue dialkylammonium salt a dimeric structure, formula II. The position marked $X$ in the formulas shown in figure 16 is occupied by $-\mathrm{COOC}_{2} \mathrm{H}_{5}$ in the case of bromo-

\footnotetext{
7 Tetrabromophenolphthalein, in contrast to tetrabromophenolsulfonephthalein (bromophenol blue), shows no visible reaction in benzene with either aromatic or aliphatic amines.
}
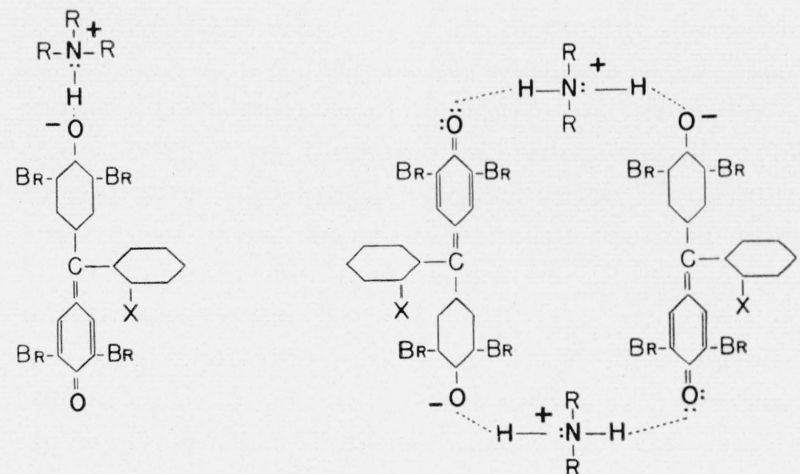

I

II

Figure 16. Formulas postulated for di- and trialkylammonium salts of bromophthalein magenta and of halogen derivatives of phenolsulfonephthalein.

I, Monomeric trialkylammonium salt; II, dimeric dialkylammonium salt. $X=-\mathrm{COOC}_{2} \mathrm{H}_{5},-\mathrm{COOC}_{4} \mathrm{H}_{9}$, or $-\mathrm{SO}_{2} \mathrm{O}-\mathrm{HNR}_{3}+$

phthalein magenta $E$ and $-\mathrm{COOC}_{4} \mathrm{H}_{9}$ in the case of bromophthalein magenta $B$. The red-purple color of solutions of the monoalkylammonium salts in benzene appears to be caused by an equilibrium between a monomer and a dimer, and the quaternary ammonium salt probably consists largely of ion-pairs. The preceding paper [1] gave reasons to support these structures. Similar explanations can be applied to the sulfonephthaleins. In the sulfonephthaleins, $X$ may be considered to represent the group $-\mathrm{SO}_{2} \mathrm{O}-\mathrm{HNR}_{3}{ }^{+}$. The lower solubility of the salts of the sulfonepthaleins in benzene is not surprising in view of their more highly polar structure.

\section{Applications of the Sulfonephthalein Indicators in Inert Solvents}

The sulfonephthaleins were developed for use in aqueous media and the sulfonic acid group, which increases their solubility in water, decreases their solubility in hydrocarbons and other inert solvents. Their salts also possess limited solubility in these solvents. Moreover, in most cases the first and second steps in their neutralization overlap somewhat. For these reasons, they are much less suitable than the bromophthalein magentas for the detection of aliphatic amines and for quantitative studies of the reactivities of amines and acids. However, the reaction of the colorless lactoid form of the sulfonephthaleins to give the yellow quinoid sulfonate can be brought about by aromatic as well as by aliphatic amines, whereas the bromo- 
phthalein magentas are not sufficiently acidic to react with aromatic amines.

While the investigations described in this paper were in progress, a colorimetric method was published for the assay of quaternary ammonium salts in dilute aqueous solutions, based upon their conversion to colored salts of bromophenol blue or bromothymol blue and the extraction of the colored salts with benzene or chlorinated hydrocarbons [21]. The low solubility of the incompletely alkylated ammonium salts of the sulfonephthaleins in inert solvents proves to be advantageous for this application of the indicators, because they remain in the aqueous layer.

The behavior of the sulfonephthaleins and their salts in other hydrocarbons and in halogenated hydrocarbons may be expected to resemble their behavior in benzene. However, in using the indicators it is well to realize that the assumption of similar modes of reaction in aqueous media and in inert solvents is not justified. Water exerts a leveling influence upon acids [22] and upon bases, and many of the common organic solvents exert a leveling effect on acids or bases or both, whereas highly specific reactions may occur in inert media, as shown in this and the preceding paper.

\section{References}

[1] Marion Maclean Davis and Priscilla J. Schuhmann, J. Research NBS 39, 221 (1947) RP1825.

[2] H. H. Cary and A. O. Beckman, J. Opt. Soc. Am. 31, 682 (1941).

[3] K. S. Gibson and M. M. Balcom, J. Research NBS 38, 601 (1947) RP1798.

[4] Terminology and symbols for use in ultraviolet, visi- ble, and infrared absorptometry, NBS Letter Cireular LC857, "(May 19, 1947).

[5] H. A. Lubs and S. F. Acree, J. Am. Chem. Soc. 38, 2772 (1916); E. C. White and S. F. Acree, 40, $1092(1918) ;$ 41, $1190(1919)$; and other papers by S. F. Acree and coworkers.

[6] I. M. Kolthoff, J. Phys. Chem. 35, 1433 (1931).

[7] R. Wizinger, Organische Farbstoffe (F. Dümmler, Berlin and Bonn, 1933).

[8] G. Schwarzenbach and G. H. Ott, Helv. Chim. Acta 20, 627 (1937), and other publications of G. Schwarzenbach and associates.

[9] G. N. Lewis, J. Franklin Inst. 226, 293 (1938).

[10] G. N. Lewis and G. T. Seaborg, J. Am. Chem. Soc. 61, 1886 (1939)

[11] G. W. Wheland, the theory of resonance and its application to organic chemistry, p. 152 (John Wiley \& Sons, Inc., New York, N. Y., 1944).

[12] W. R. Orndorff and F. W. Sherwood, J. Am. Chem. Soc. 45, 486 (1923.)

[13] B. Cohen, Reprint No. 1131 from the Public Health Reports (Government Printing Office, Washington, D. C., 1927)

[14] W. C. Harden and N. L. Drake, J. Am. Chem. Soc. 51, $562(1929)$.

[15] W. R. Orndorff and A. C. Purdy, J. Am. Chem. Soc. 48, 2212 (1926).

[16] H. A. Lubs and W. Mi. Clark, J. Wash. Acad. Sci. 6, 481 (1916).

[17] W. R. Orndorff and R. T. K. Cornwell, J. Am. Chem. Soc. 48, 981 (1926).

[18] V. K. LaMier and H. C. Downes, J. Am. Chem. Soc. 55, 1854 (1933); Chem. Reviews 13, 47 (1933).

[19] D. C. Griffiths, J. Chem. Soc. (London), 818 (1938).

[20] J. N. Brønsted, Ber. deut. chem. Ges. 61, 2049 (1928).

[21] Mi. E. Auerbach, Ind. Eng. Chem., Anal. Ed. 15, 492 (1943) ; 16, 739 (1944). See also E. L. Colichman, Anal. Chem. 19, 430 (1947).

[22] A. Hantzsch, Z. Elektrochem. 29, 221 (1923); 30, 194 (1924).

Washington, April 13, 1948. 